PROCEEDINGS OF THE

AMERICAN MATHEMATICAL SOCIETY

Volume 128, Number 3, Pages 919-922

S 0002-9939(99)05068-6

Article electronically published on May 6, 1999

\title{
SYNCHRONISM OF AN INCOMPRESSIBLE NON-FREE SEIFERT SURFACE FOR A KNOT AND AN ALGEBRAICALLY SPLIT CLOSED INCOMPRESSIBLE SURFACE IN THE KNOT COMPLEMENT
}

\author{
MAKOTO OZAWA \\ (Communicated by Ronald A. Fintushel)
}

\begin{abstract}
We give a necessary and sufficient condition for knots to bound incompressible non-free Seifert surfaces.
\end{abstract}

\section{INTRODUCTION}

A Seifert surface for a knot in the 3-sphere is said to be free (unknotted) if the fundamental group of its complement is free, otherwise non-free (knotted). We note that any knot bounds both free and non-free Seifert surfaces if one admits Seifert surfaces to be compressible. For this reason, hereafter we consider only incompressible Seifert surfaces. Hatcher and Thurston classified incompressible surfaces in 2-bridge knot complements [3]. This result implies that any incompressible Seifert surface for 2-bridge knots is free. However, there exist knots which bound both free and non-free incompressible Seifert surfaces ([13], [10]). Moreover, there exist knots which bound only non-free incompressible Seifert surfaces ([9], [7]). In [6], C. H. Giffen and L. C. Siebenmann raised the following problem.

Problem 1.1 ([6, Problem 1.20 (B)]). Which knots bound an incompressible free Seifert surface?

In this paper, we give a necessary and sufficient condition for knots to bound incompressible non-free Seifert surfaces. As its corollary, we get a necessary condition for knots to bound incompressible free Seifert surfaces.

Let $K$ be a knot in the 3 -sphere $S^{3}$. For a closed surface $S$ in $S^{3}-K$, we define the order $o(S ; K)$ of $S$ for $K$ as follows. Let $i: S \rightarrow S^{3}-K$ be the inclusion map and let $i_{*}: H_{1}(S) \rightarrow H_{1}\left(S^{3}-K\right)$ be the induced homomorphism. Since $\operatorname{Im}\left(i_{*}\right)$ is a subgroup of $H_{1}\left(S^{3}-K\right)=\mathbb{Z}\langle$ meridian $\rangle$, there is an integer $m$ such that $\operatorname{Im}\left(i_{*}\right)=m \mathbb{Z}$. Then we define $o(S ; K)=m$.

Theorem 1.2. Let $K$ be a knot in $S^{3}$. Then the following conditions are equivalent.

(1) There exists an incompressible non-free Seifert surface $F$ for $K$.

(2) There exists a closed incompressible surface $S$ in $S^{3}-K$ with $o(S ; K)=0$.

Received by the editors June 18, 1997 and, in revised form, May 4, 1998.

1991 Mathematics Subject Classification. Primary 57M25.

Key words and phrases. Seifert surface, closed incompressible surface.

(c)1999 American Mathematical Society 
Moreover, if these conditions hold, then we can take $F$ and $S$ so that they are disjoint.

For knots in $S^{3}$ whose complements contain no incompressible and meridionally incompressible closed surfaces, any closed incompressible surface in exteriors of those knots has a compressing disk in $S^{3}$ which intersects the knots in one point. Hence the closed incompressible surface has the order \pm 1 .

Corollary $1.3([1],[8])$. Let $K$ be a toroidally alternating knot or closed 3-braid knot in $S^{3}$. Then any incompressible Seifert surface for $K$ is free.

Remark 1.4. Toroidally alternating knots ([1]) include alternating knots ([11]), almost alternating knots ([2]) and Montesinos knots ([12]).

\section{ProOF}

All manifolds are assumed to be compact and orientable.

Lemma 2.1 ([5, IV.5, IV.10]). Let $M$ be a 3-manifold and $F$ an incompressible surface properly embedded in $M$. Put $M^{\prime}=c l(M-N(F))$. Then:

(1) $M$ is irreducible if and only if $M^{\prime}$ is irreducible.

(2) Any closed incompressible surface embedded in int $M^{\prime}$ is incompressible in $M$.

Proof. (1) Suppose that $M$ is irreducible and $M^{\prime}$ is reducible. Let $S$ be a reducing 2 -sphere for $M^{\prime}$. Then $S$ bounds a 3-ball $B$ in $M$ and $F$ is contained in $B$. Hence $F$ is a closed orientable surface, and hence $\pi_{1}$-injective in $B$. Therefore $F$ is a 2 -sphere and bounds a 3 -ball in $B$, hence in $M$. This contradicts the incompressibility of $F$ in $M$.

Suppose that $M$ is reducible and $M^{\prime}$ is irreducible. Let $S$ be a reducing 2-sphere for $M$. We may assume that $S \cap F$ consists of loops. Let $D$ be an innermost disk in $S$. Since $F$ is incompressible in $M, \partial D$ bounds a disk $D^{\prime}$ in $F$. Since $M^{\prime}$ is irreducible, the 2-sphere $D \cup D^{\prime}$ bounds a 3 -ball $B$ in $M^{\prime}$. By using the 3 -ball $B$, we can reduce $|S \cap F|$. Hence we may assume that $S \cap F=\emptyset$. Thus $S$ is contained in $M^{\prime}$ and bounds a 3-ball in $M^{\prime}$, hence in $M$. This contradicts the supposition.

(2) Suppose that there exists a closed incompressible surface $S$ properly embedded in $M^{\prime}$ which is compressible in $M$. Let $D$ be a compressing disk for $S$ in $M$. We may assume that $D \cap F$ consists of loops. Let $d$ be an innermost disk in $D$. Since $F$ is incompressible in $M, \partial d$ bounds a disk $d^{\prime}$ in $F$. Let $d^{\prime \prime}$ be an innermost disk in $d^{\prime}$. By cutting and pasting $D$ along $d^{\prime \prime}$, we get a compressing disk for $S$ in $M$ with fewer intersections with $F$ than $D$ and a 2-sphere. By induction on $|D \cap F|$, we may assume that $D \cap F=\emptyset$. Thus $D$ is contained in $M^{\prime}$. This contradicts the supposition.

Lemma $2.2([4,5.2],[5$, IV.15]). Let $M$ be an irreducible 3-manifold with connected boundary. Then the following conditions are mutually equivalent.

(1) $\pi_{1}(M)$ is free.

(2) $M$ is a handlebody.

(3) $M$ does not contain any closed incompressible surface.

Proof. (1) $\rightarrow(3)$. Suppose that $M$ contains a closed incompressible surface $S$. Since $S$ is 2-sided in $M, S$ is $\pi_{1}$-injective in $M$. Therefore $\pi_{1}(S)$ is isomorphic to a subgroup of $\pi_{1}(M)$. Since any subgroup of a free group is free, $\pi_{1}(S)$ is free. Hence 
$S$ is a 2 -sphere. By the irreducibility of $M, S$ bounds a 3 -ball in $M$. This contradicts the supposition.

$(3) \rightarrow(2)$. We will show this by induction on $g(\partial M)$. If $g(\partial M)=0, M$ is a 3 -ball because $M$ is irreducible. Suppose that $(3) \rightarrow(2)$ of Lemma 2.2 holds if $g(\partial M)<g$, and suppose that $g(\partial M)=g$. Since $M$ does not contain any closed incompressible surface, $\partial M$ is compressible in $M$. Let $D$ be a compressing disk for $\partial M$ in $M$. Put $M^{\prime}=\operatorname{cl}(M-N(D))$. Then by Lemma $2.1, M^{\prime}$ is irreducible and does not contain any closed incompressible surface. By the supposition of induction, each component of $M^{\prime}$ is a handlebody. Hence $M$ is a handlebody.

$(2) \rightarrow(1)$. By the definition of a handlebody.

Proof of Theorem 1.2 (1) $\rightarrow$ (2). Suppose (1) of Theorem 1.2. Let $F$ be an incompressible non-free Seifert surface for $K$. Put $M=\operatorname{cl}\left(S^{3}-N(K)\right)$ and isotop $F$ so that $F \cap N(K)$ is an annulus. We also denote $F \cap M$ by $F$. Since $\pi_{1}\left(S^{3}-F\right) \cong$ $\pi_{1}(\operatorname{cl}(M-N(F)))$ is not free, by Lemma 2.2 , it contains a closed incompressible surface $S$. By Lemma $2.1(2), S$ is also incompressible in $M$, hence in $S^{3}-K$. We take $2 g(S)$ simple loops $l_{1}, \ldots, l_{2 g(S)}$ on $S$ so that $H_{1}(S)=\mathbb{Z}\left\langle l_{1}\right\rangle \oplus \cdots \oplus \mathbb{Z}\left\langle l_{2 g(S)}\right\rangle$. Since $S \cap F=\emptyset, l k\left(l_{i}, K\right)=0(i=1, \ldots, 2 g(S))$, thus $i_{*}\left(\left\langle l_{i}\right\rangle\right)=0$, where $i_{*}$ denotes the induced homology homomorphism as in the definition of order. Hence $i_{*}\left(H_{1}(S)\right)=0$ and we have condition (2).

Proof of Theorem 1.2 (2) $\rightarrow$ (1). Suppose (2) of Theorem 1.2.

Claim 2.3. There exists a Seifert surface $F$ for $K$ such that $F \cap S=\emptyset$.

Proof. Let $F$ be any Seifert surface for $K$. Let $G$ be a graph with one vertex $v$ and $2 g(S)$ edges $e_{1}, \ldots, e_{2 g(S)}$ embedded in $S$ such that $S-G$ is an open disk. We may assume that $F$ intersects $S$ and $G$ transversely and does not intersect $v$. Give an orientation to $F$ and the edges of $G$ arbitrarily. Suppose that $F \cap G \neq \emptyset$. Then there exists a pair of points $p_{1}$ and $p_{2}$ of $F \cap G$ such that $p_{1}$ and $p_{2}$ are adjacent in an edge $e_{i}$ and have intersection numbers +1 and -1 respectively. Indeed, $l k\left(e_{i}, K\right)=0$ since $o(S ; K)=0$. Let $a$ be a subarc of $e_{i}$ bounded by $p_{1}$ and $p_{2}$. By tubing $F$ along $a$, we get a Seifert surface for $K$ with fewer intersections with $G$ than $F$. By induction on $|F \cap G|$, we may assume that $F \cap G=\emptyset$. Since $S-G$ is an open disk, by an innermost loop, and cut and paste argument, we can get a Seifert surface $F$ for $K$ with $F \cap S=\emptyset$.

Claim 2.4. There exists an incompressible Seifert surface $F$ for $K$ such that $F \cap S=$ $\emptyset$.

Proof. By Claim 2.3, there exists a Seifert surface $F$ for $K$ such that $F \cap S=\emptyset$. Suppose that $F$ is compressible. Let $D$ be a compressing disk for $F$ in $S^{3}-K$. By the argument which is similar to the proof of Lemma 2.1 (2), we may assume that $D \cap S=\emptyset$. Now we compress $F$ along such $D$. Then by ignoring a closed component, we get a Seifert surface for $K$ such that it does not intersect $S$ and has fewer genus than $F$. By an induction on $g(F)$, we can get an incompressible Seifert surface $F$ for $K$ such that $F \cap S=\emptyset$.

By Claim 2.4, there exists an incompressible Seifert surface $F$ for $K$ such that $F \cap S=\emptyset$. Then $S$ is a closed incompressible surface in $\operatorname{cl}\left(S^{3}-N(F)\right)$. Hence by Lemma 2.2, $\pi_{1}\left(c l\left(S^{3}-N(F)\right)\right)$ is not free. Thus $F$ is an incompressible non-free Seifert surface for $K$. 


\section{REFERENCES}

[1] C. Adams, Toroidally alternating knots and links, Topology 33, (1994), 353-369. MR 95e:57006

[2] C. Adams, J. Brock, J. Bugbee, T. Comar, K. Faigin, A. Huston, A. Joseph and D. Pesikoff, Almost alternating links, Topology and its Appl. 46, (1992), 151-165. MR 93h:57005

[3] A. Hatcher and W. Thurston, Incompressible surfaces in 2-bridge knot complements, Invent. Math. 79, (1985), 225-246. MR 86g:57003

[4] J. P. Hempel, 3-Manifolds, Volume $\mathbf{8 6}$ of Ann. of Math. Stud., Princeton Univ. Press, 1976. MR 54:3702

[5] W. H. Jaco, Lectures on Three-manifold Topology, Volume 43 of CBMS Regional Conf. Ser. in Math., American Math. Soc., 1980. MR 81k:57009

[6] R. Kirby, Problems in low-dimensional topology, Part 2 of Geometric Topology (ed. W. H. Kazez), Studies in Adv. Math., Amer. Math. Soc. Inter. Press, 1997. MR 80g:57002

[7] M. Kobayashi and T. Kobayashi, On canonical genus and free genus of knot, J. Knot Theory and its Ramifi. 5, (1996), 77-85. MR 97d:57008

[8] M. T. Lozano and J. H. Przytycki, Incompressible surface in the exterior of a closed 3-braid, Math. Proc. Camb. Phil. Soc. 98, (1985), 275-299. MR 87a:57013

[9] H. C. Lyon, Knots without unknotted incompressible spanning surfaces, Proc. Amer. Math. Soc. 35, (1972), 617-620. MR 46:2663

[10] H. C. Lyon, Simple knots without unique minimal surfaces, Proc. Amer. Math. Soc. 43, (1974), 449-454. MR 51:14019

[11] W. Menasco, Closed incompressible surfaces in alternating knot and link complements, Topology Vol. 23, No. 1, (1984) 37-44. MR 86b:57004

[12] U. Oertel, Closed incompressible surfaces in complements of star links, Pacific J. of Math., 111, (1984), 209-230. MR 85j:57008

[13] C. B. Schaufele, The commutator group of a doubled knot, Duke Math. J. 34, (1967), 677-681. MR 35:7328

Department of Science, School of Education, Waseda University, 1-6-1 Nishiwaseda, SHINJUKU-KU, TOKYO 169-8050, JAPAN

E-mail address: ozawa@m.waseda.ac.jp 